\title{
Sara - A Semi-Automatic Framework for Social Network Analysis
}

\author{
Carlos Barbosa \\ Universidade Federal de São João del-Rei \\ Rodovia BR-494, s/n, São João Del Rei, Minas Gerais \\ carlosmagno@outlook.com \\ Vinícius Vieira \\ Universidade Federal de São João del-Rei \\ Rodovia BR-494, s/n, São João Del Rei, Minas Gerais \\ vinicius@ufsj.edu.br
}

\begin{abstract}
Understanding the dissemination of information in social networks has become essential for modern societies. These networks have dramatically changed the mode of communication, relationship, marketing, and access to information. Platforms such as Twitter, and WhatsApp are some representatives of these new information propagation media that represented a major shift in a model centered on traditional communication vehicles. This new decentralized environment gave voice to marginalized groups, riots such as the Arab Spring, growth of populist parties and false news waves across the globe. Therefore, considering the influence of these platforms in several aspects of society, this work presents a framework for characterizing the diffusion of information in social networks, especially on Twitter. This characterization is accomplished through the use of complex network and text mining techniques, exploring the generation of a retweets network, the formation of communities around specific users, cascades of information, analysis of feelings and modeling of topics. As an evaluation this model is applied in characterizing a network of retweets generated around the discussion of pension reform of Brazil on Twitter.
\end{abstract}

\section{KEYWORDS}

social network, data mining, complex network, information cascade

\section{INTRODUÇÃO}

Com o surgimento da internet e posteriormente da web e das redes sociais, como Twitter, o modo de consumo de notícias, entretenimento, marketing, relacionamento e até mesmo os processos eleitorais se alteraram drasticamente. Essas redes tiveram uma grande participação em diversos processos políticos pelo mundo como as eleições de 2018 no Brasil, a corrida presidencial dos Estados Unidos de 2016 [2], o processo do referendo do Brexit no Reino Unido ${ }^{1}$ e o crescimento de candidatos e partidos que abraçam o populismo no mundo[4, 12]. Estas plataformas também estiveram presentes em grandes protestos como na articulação da primavera árabe[8] no Oriente Médio e no Norte da África em 2011, nos protestos de 2013

\footnotetext{
${ }^{1}$ https://www.dw.com/pt-br/pivô-do-caso-facebook-acusa-manipulaç ao-no-brexit/ a- 43152832
}

In: XVI Workshop de Trabalhos de Iniciação Científica (WTIC 2019), Rio de Janeiro, Brasil. Anais Estendidos do Simpósio Brasileiro de Sistemas Multimídia e Web (WebMedia). Porto Alegre: Sociedade Brasileira de Computação, 2019.

ISSN 2596-1683

\author{
Lucas Félix \\ Universidade Federal de São João del-Rei \\ Rodovia BR-494, s/n, São João Del Rei, Minas Gerais \\ lucasgsfelix@gmail.com \\ Carolina Xavier \\ Universidade Federal de São João del-Rei \\ Rodovia BR-494, s/n, São João Del Rei, Minas Gerais \\ carolinaxavier@ufsj.edu.br
}

no Brasil [5] e na greve dos caminhoneiros de 2018 [13]. Também podemos destacar o papel destas plataformas para a propagação de boatos relacionando vacinas ao autismo [7].

Portanto, devido à grande relevância das redes sociais na sociedade moderna, a caracterização e monitoração do conteúdo que se propaga nesta rede é de grande importância para a sociedade e para o poder público. Podemos encontrar na literatura trabalhos como Soroush, Haewoon e Resende[9, 11, 17] que se debruçam nessa caracterização e monitoração das redes sociais. Desde modo, este trabalho apresenta um framework que permite uma análise semiautomatizada do espalhamento de informações em redes sociais em especial no Twitter. O framework desenvolvido combina redes complexas com mineração de texto para monitorar o espalhamento de informação sobre determinado assunto, permitindo encontrar comunidades formadas em torno de determinados usuários e monitorar o sentimento envolvido.

Com o intuito de avaliar esta metodologia, foi realizada a coleta de mensagens relacionadas a reforma da previdência no Twitter no período de Fevereiro de 2019. Foram coletados 120 mil tweets que deram origem a uma rede com mais de 40 mil vértices e 80 mil arestas. Além disso, foram coletados 70 mil comentários postados em mensagens relacionadas a previdência. Como resultado, foi encontrado que o principal sentimento em torno das discussões é negativo, e que grupos pró-reforma estavam mais ativos no período de coleta, sendo que foi possível identificar uma grande utilização de termos agressivos.

\section{TRABALHOS RELACIONADOS}

O trabalho de Soroush[17] apresenta um estudo a respeito da caracterização do espalhamento de notícias verdadeiras e falsas no Twitter. Neste estudo é caracterizado o espalhamento de aproximadamente 126 mil tweets que foram retuitados mais de 4 milhões de vezes. Os mesmos são classificados em verdadeiros, falsos ou mistos, com base em seis checadores de notícias independentes. Através deste estudo eles concluíram que as notícias verdadeiras e falsas se espalham de maneira distinta, sendo que as notícias falsas se propagam mais rapidamente que as informações verdadeiras. Inclusive destacam que as emoções que as mesmas provocam nos usuários são distintas, e que as notícias falsas sobre política são as que apresentam uma maior capacidade de viralização, seguida das informações sobre terrorismo. Por meio deste estudo também chegaram à conclusão que as notícias falsas são propagadas principalmente por usuários reais e que estas notícias passam uma ideia 
de serem mais novas e que estão sendo ocultas pelos veículos de mídia tradicionais, tal efeito é indicado como um forte indicador para viralização destas notícias. A principal correlação deste trabalho com o desenvolvido nesta pesquisa está no estudo do espalhamento de informações. Além disso, este trabalho apresenta uma análise profunda do conteúdo dos tweets que foram mais propagados.

O trabalho de Souza [15] apresenta uma avaliação de diferentes classificadores para identificação de sentimento em textos curtos em português. No trabalho, é apresentado uma comparação entre Support Vector Machine (SVM), Multinominal Naive bayes (MNB) e Max Entropy (ME) aplicados no caso de uso de identificação de tweets a favor ou contra o impeachment de 2016 no Brasil.

Tsugawa[16] analisa a correlação entre o sentimento e o espalhamento de informação no Twitter do Japão. Neste trabalho são analisados cerca de 4 milhões de tweets coletados em julho de 2013. Os tweets coletados foram classificados em relação ao sentimento em neutro, negativo, positivo com base em um dicionário com a polarização das palavras chamado $\mathrm{MrCab}$. No final chegaram à conclusão que tweets com sentimento negativo possuem uma capacidade de viralização superior à mensagens classificadas como positiva e neutras.

Resende[11] apresenta um modelo de monitoração de redes sociais em especial do Whatsapp no qual são monitorados a opinião de 127 grupos públicos relacionados a discussões políticas e de notícias, em geral. Através desta monitoração dos grupos públicos foram coletadas cerca de 210 mil mensagens no período de $27 / 04$ a 30/05 de 2018. As palavras mais utilizadas nos grupos foram sumarizadas por meio da utilização de uma nuvem de palavras. Os grupos coletados foram classificados em 8 categorias, sendo as mesmas representadas por Debates, Direita, Ideologias, Notícias, Partidos, Pró-Bolsonaro e Pro-Lula, sendo que o principal conteúdo propagado nestes grupos foram imagens e o menor áudio. A principal similaridade deste trabalho com o desenvolvido está na caracterização das notícias propagadas em grupos das redes sociais.

Todos os trabalhos relacionados caracterizam o espalhamento de informação sendo que os mesmos se diferenciam nas técnicas utilizadas.

\section{METODOLOGIA}

Uma visão geral da metodologia pode ser observada na figura 1. O framework desenvolvido pode ser resumido em três módulos essenciais. Sendo um módulo responsável pela coleta, o segundo pela análise, quanto às técnicas de redes complexas, focado na geração da rede de espalhamento de informação e detecção de comunidades. O terceiro módulo, utiliza técnicas de mineração de texto no qual todo o conteúdo coletado é sumarizado e exibido como uma nuvem de palavras, nesta etapa o sentimento em torno da discussão também é aferido utilizando uma abordagem léxica. Os módulos são apresentados a seguir: I) Coleta, Armazenamento e Limpeza: A coleta dos dados é realizada por meio da Application Programming Interface(API) do Twitter, no qual são coletados todos os Tweets e retweets sobre determinado assunto que o usuário considere importante, e por meio de um crawler de comentários. O módulo de coleta de comentários recupera e coleta de todos os comentários postados nas mensagens capturadas pela API. Após o processo de coleta as mensagens são armazenadas e passam por um processo

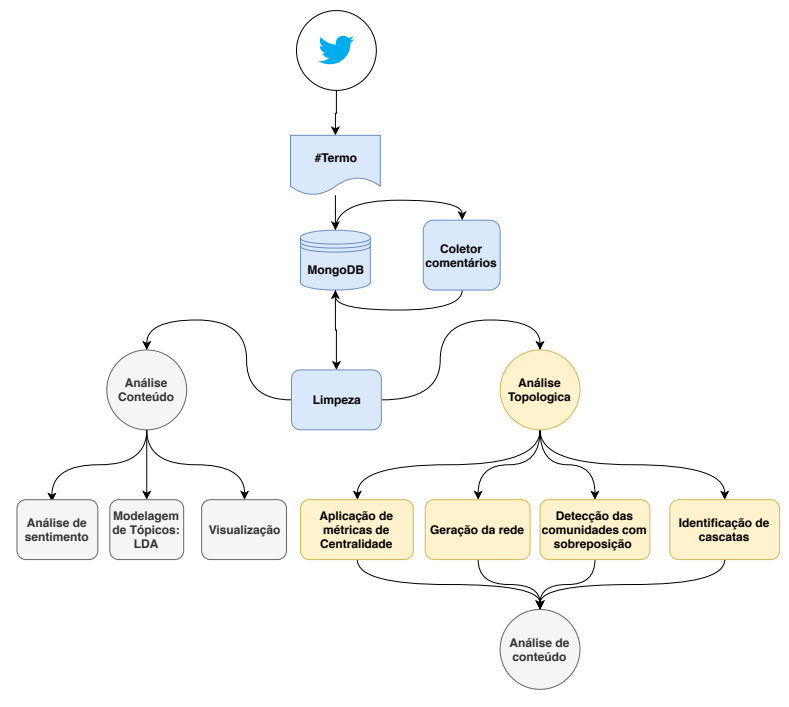

Figura 1: Visão geral metodologia

de limpeza, neste processo são eliminadas stopwords, termos que não agregam muita informação no texto, hashtags, emojis, números, links, imagens, menções a usuários e palavras quebradas, termos com um carácter, caracteres especiais e acentuação. Todos os dados coletados e limpos são armazenados em um banco de dados não relacional no formato favaScript Object Notation(JSON). II) Análise Estrutural: Este módulo realiza a geração de uma rede direcionada sem peso nas arestas de retweets, na qual os vértices são perfis que postaram determinada mensagem sobre um assunto e as arestas indicam os perfis que realizaram o retweet desta mensagem. Após a geração da rede, este método aplica métricas de centralidade com o intuito de encontrar os vértices de maior importância e detecta as comunidades formadas em torno destes vértices. Nesta etapa, também é realizado uma análise de formação de cascatas de espalhamento de informação, na qual é utilizado uma abordagem apresentada por Schons[14], na qual é gerada uma árvore de propagação através de um modelo de espalhamento de informação, utilizando Independent Cascade Model(ICM), e na geração de um nome canônico das árvores, com o intuito de comparar as árvores e encontrar os padrões frequentes. III) Análise de Conteúdo: Com o intuito de complementar análise topológica este módulo realiza uma sumarização do conteúdo, análise de sentimento e visualização dos dados. Os tweets, comentários e retweets coletados são sumarizados por meio da aplicação de técnica de modelagem de tópicos, especificamente o Latent Dirichlet Allocation (LDA), no qual uma grande quantidade de documentos podem ser representados em um conjunto de tópicos e termos resumindo toda a informação coletada [3]. Com o intuito de permitir uma análise rápida os 10 tópicos mais relevantes da LDA, foram transformados em nuvem de palavras, considerando a frequência de aparecimento dos termos. Os termos coletados também foram submetidos a uma análise de sentimento utilizando uma abordagem léxica por meio da ferramenta de análise de sentimento de tweets em português LeIA [1] uma adaptação do metodologia Vader [6]. Nessa abordagem 
cada palavra já foi previamente classificada e estabelecido um sentimento negativo ou positivo. Este módulo é aplicado para uma visão geral da informação quanto os tweets, retweets, comentários e uma visão específica focada na análise de determinadas comunidades formadas em torno de determinados perfis considerados relevantes.

\subsection{Algoritmo de Detecção de Comunidades com Sobreposição}

A detecção de comunidades é realizada através da modificação do algoritmo de Lancichinetti [10], no qual as sementes utilizadas para detecção das comunidades são definidas de acordo com a aplicação das métricas de centralidade. $\mathrm{Na}$ abordagem original, essa escolha de vértices era realizada de maneira aleatória. Por meio desta nova abordagem é possível encontrar as comunidades que se formam em torno de um vértice específico, considerando algum critério. Esse critério pode ser de acordo com a relevância de determinado perfil fora da rede social. O processo de detecção de cluster em torno de um vértice pode ser resumido com os seguintes passos: I) escolha um vértice A de acordo com ranking de centralidade; II) detecte a comunidade em torno do vértice A; III) escolha um vértice $B$ de acordo com ranking de centralidade; IV) detecte a comunidade de $\mathrm{B}$, explorando todos os vértices, inclusive os que já estão em uma comunidade; V) repita o processo do item III. A detecção de comunidades natural em torno de cada vértice é realizada através da maximização da função de fitness, apresentada por Lancichinetti[10]. Destacamos que este algoritmo é flexível para se utilizar outro critério que o usuário considera interessante, para se encontrar as ego comunidades.

\section{EXPERIMENTOS E DISCUSSÕES}

Considerando a base de 120 mil tweets relacionados à previdência, foi possível se gerar uma rede de 41665 vértices e 80776 arestas. Sendo que as análises foram realizadas na componente gigante que possui 40031 vértices e 79776 arestas com um grau médio de entrada e saída de 1.992. Quanto a distribuição de grau a rede segue uma distribuição com cauda longa. Portanto, poucos usuários possuem tweets que sofreram vários retweets. O grau máximo de entrada foi 140 e o grau máximo de saída foi 2721. Sendo os perfis vinculados aos Presidenciáveis nas eleições do Brasil em 2018 Guilherme Boulos e João Amoedo com os maiores graus de saída.

Com o intuito de encontrar os elementos de maior importância quanto à discussão da reforma da previdência na rede, apresentamos os resultados encontrados com aplicação das métricas de centralidade. Podemos observar um resumo dos resultados encontrados a seguir: I) Betweenness: Mariada94156889, DilmaResiste. II) PageRank: poirrot, ma_achaves; III) Degree: GuilhermeBoulos, joaoamoedonovo ; IV) Curtidas: jairbolsonaro, joaoamoedonovo; V) Retweets: jairbolsonaro, joaoamoedonovo;

Considerando a centralidade por Betweenness, os perfis de maior importância são críticos a reforma. Por sua vez, considerando a abordagem de Curtidas e Retweets, os perfis de maior importância são pró-reforma. Realizando uma análise dos perfis foi possível identificar que na sua grande maioria os perfis destacados possuem importância política fora da rede social.

Aplicando a identificação de padrões de espalhamento foi possível observar que não ocorreu a formação de grandes cascatas, sendo o padrão mais comum o que representa somente o retweet da mensagem original.

Com o intuito de ter uma visão geral do conteúdo que está sendo propagado na rede apresentamos os resultados da análise dos comentários e tweets sumarizados com aplicação da LDA e da nuvem de palavras. Podemos observar na Figura 2 os principais pontos de discussão relacionados aos tweets e retweets.

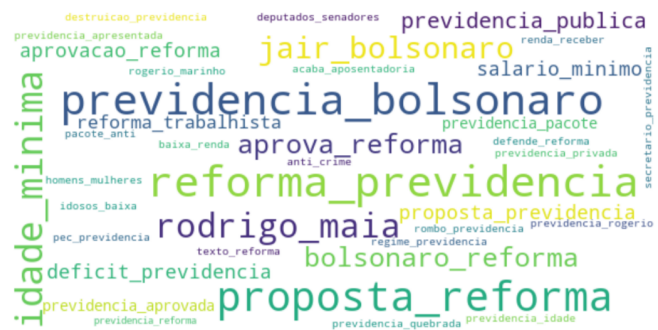

Figura 2: Tópicos considerando Tweets e retweets

Observando os tópicos destacados é possível identificar a presença de termos destacando a apresentação da proposta da reforma, alteração no regime da previdência, previdência privada, salário mínimo, idade mínima, deficit da previdência, destruição da previdência, deputados e senadores. Isso demonstra uma ampla discussão em torno dos principais pontos de alterações da reforma. Além disso, é possível observar uma discussão relacionada a reforma trabalhista, pacote anticrime e referências ao Presidente da República e da Câmara. Podemos destacar que a discussão em torno da previdência acaba englobando outros projetos do governo.

Considerando os comentários postados pelos usuários em tweets e retweets relacionados à previdência, foi possível encontrar os tópicos apresentados na Figura 3.

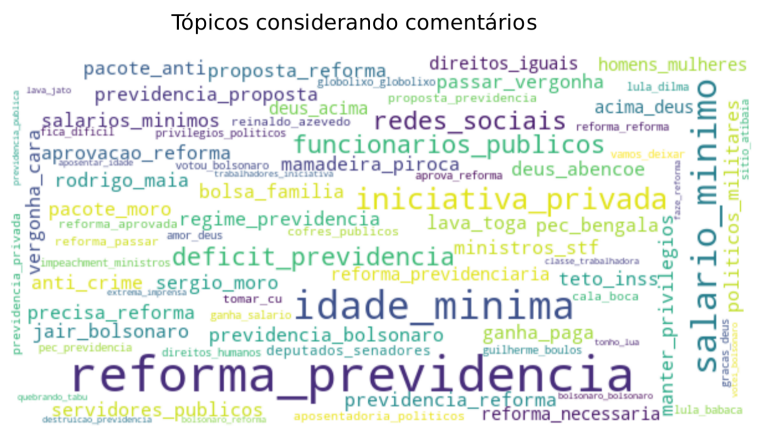

Figura 3: Principais tópicos considerando comentários

É possível observar que os comentários no geral são um pouco mais agressivos, considerando a utilização de termos de ataques à imprensa e ao judiciário. Também podemos destacar a presença de termos que fazem referência a fake news, alterações no regime da previdência e referências a ministros do Superior Tribunal Federal.

Aplicando a análise de sentimento léxica nos tweets e nos comentários, encontramos que o sentimento envolvido na discussão da previdência é negativo. 


\subsection{Análise Comparativa de Perfis}

Com intuito de explorar as características dos vértices presentes nas comunidades foram selecionados dois vértices e suas respectivas comunidades. Estes vértices foram escolhidos a partir da sua importância, considerando a métrica de centralidade por grau e as suas posições em relação à reforma da previdência. Os vértices selecionados pertencem ao perfil dos presidenciáveis da eleição para Presidente em 2018, João Amoedo e Guilherme Boulos, sendo um favorável e outro contrário à proposta. Foi aplicada uma análise de conteúdo extraindo os principais tópicos gerados a partir dos comentários de usuários dentro dessas comunidades.

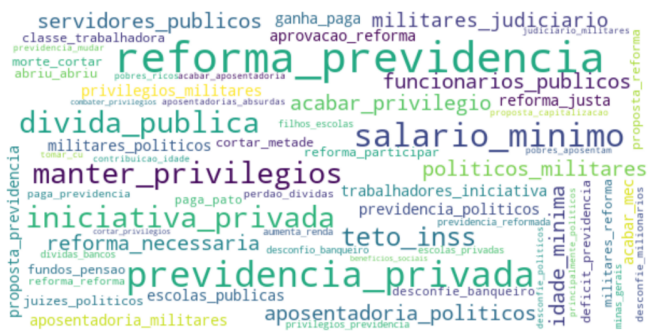

Figura 4: Comentários comunidade João Amoedo

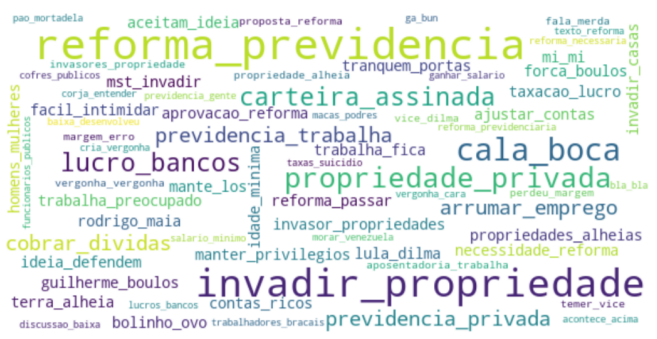

Figura 5: Comentários comunidade do Guilherme Boulos

Observando os resultados apresentados na Figura 5 quanto a Guilherme Boulos, podemos observar que os principais tópicos referentes aos comentários em suas postagens são de ataques, e não diretamente relacionados à previdência. Por sua vez, analisando os tópicos em destaque de João Amoedo 4, a maioria dos comentários são pró-reforma diretamente relacionados à previdência.

Realizando uma análise de busca de padrões de cascata dentro das comunidades, os formatos mais encontrados foram os padrões de uma única camada. Indicando que praticamente não ocorre a formação de cascatas.

Por meio dessa breve exploração foi possível observar que perfis pró-reforma estão mais ativos inclusive comentando postagens dentro da comunidade de propagação de usuários contrários a reforma.

\section{CONCLUSÃO E TRABALHOS FUTUROS}

Este trabalho apresentou a construção de um framework que permite uma coleta e análise semi-automatizada do espalhamento de notícias em redes sociais, combinando redes complexas e mineração de texto. Como resultado, este trabalho foi capaz de caracterizar o espalhamento de mensagens relacionadas à reforma da previdência no Twitter em fevereiro de 2019. Apresentando que a rede gerada através dos retweets não apresenta a formação de grandes cascatas de informação e que a rede apresenta uma distribuição de grau de cauda longa. Foi possível identificar que usuários pró-reforma estavam mais ativos no período de coleta, sendo responsáveis inclusive por uma grande parte de comentários em perfis críticos a reforma. Também foi possível identificar a utilização de termos mais radicais por parte de usuários contra órgãos de imprensa e ao judiciário, e que dentro da discussão da reforma outros projetos do governo também estavam sendo discutidos. Além do mais, foi possível identificar que o principal sentimento relacionado à reforma é negativo.

Como trabalho futuro podemos destacar o desenvolvimento de um módulo de identificação de perfis falsos e uma interface web permitindo o fácil acesso de jornalistas de pequenos veículos de mídia.

\section{REFERÊNCIAS}

[1] Rafael J. A. Almeida. 2018. LeIA - Léxico para Inferência Adaptada. https: //github.com/rafjaa/LeIA.

[2] Alessandro Bessi and Emilio Ferrara. 2016. Social bots distort the 2016 US Presidential election online discussion. (2016).

[3] David M Blei, Andrew Y Ng, and Michael I Jordan. 2003. Latent dirichlet allocation. fournal of machine Learning research 3, Jan (2003), 993-1022.

[4] Jamil Chade. 2018. Populistas usam redes sociais para disseminar ódio, alerta ONU - Internacional. https://internacional.estadao.com.br/noticias/geral, populistas-usam-redes-sociais-para-disseminar-odio-alerta-onu, 70002579324

[5] Camilla Costa. 2013. Brasileiros 'descobrem' mobilização em redes sociais durante protestos - BBC News Brasil. https://www.bbc.com/portuguese/noticias/2013/ 07/130628_protestos_redes_personagens_cc

[6] CJ Hutto Eric Gilbert. 2014. Vader: A parsimonious rule-based model for sentiment analysis of social media text. In Eighth International Conference on Weblogs and Social Media (ICWSM-14). Available at (20/04/16) http://comp. social. gatech. edu/papers/icwsm14. vader. hutto. pdf.

[7] Victor Grech. 2017. Fake news and post-truth pronouncements in general and in early human development. Early Human Development 115 (2017), 118 - 120. https://doi.org/10.1016/j.earlhumdev.2017.09.017

[8] Philip N Howard, Aiden Duffy, Deen Freelon, Muzammil M Hussain, Will Mari, and Marwa Maziad. 2011. Opening closed regimes: what was the role of social media during the Arab Spring? Available at SSRN 2595096 (2011).

[9] Haewoon Kwak, Changhyun Lee, Hosung Park, and Sue Moon. 2010. What is Twitter, a social network or a news media?. In Proceedings of the 19th international conference on World wide web. AcM, 591-600.

[10] Andrea Lancichinetti, Santo Fortunato, and János Kertész. 2009. Detecting the overlapping and hierarchical community structure in complex networks. New Journal of Physics 11, 3 (2009), 033015.

[11] Gustavo Resende, Johnnatan Messias, Márcio Silva, Jussara Almeida, Marisa Vasconcelos, and Fabrício Benevenuto. 2018. A System for Monitoring Public Political Groups in WhatsApp. In Proceedings of the 24th Brazilian Symposium on Multimedia and the Web. ACM, 387-390.

[12] Rojo. 2019. Análise | Os novos populistas se aproveitam da necessidade de reconhecimento. https://brasil.elpais.com/brasil/2019/01/04/opinion/1546605756_ 247286.html

[13] Amanda Rossi. 2018. Como o WhatsApp mobilizou caminhoneiros, driblou governo e pode impactar eleições - BBC News Brasil. https://www.bbc.com/ portuguese/brasil-44325458

[14] Thiago Schons. 2015. Análise de Padrões em Espalhamento Gerado por Modelos. (2015).

[15] Bruno A Souza, Thais G Almeida, Alice AF Menezes, Fabíola G Nakamura, Carlos Figueiredo, and Eduardo F Nakamura. 2016. For or against?: Polarity analysis in tweets about impeachment process of brazil president. In Proceedings of the 22nd Brazilian Symposium on Multimedia and the Web. ACM, 335-338.

[16] Sho Tsugawa and Hiroyuki Ohsaki. 2015. Negative Messages Spread Rapidly and Widely on Social Media. In Proceedings of the 2015 ACM on Conference on Online Social Networks (COSN '15). ACM, New York, NY, USA, 151-160. https: //doi.org/10.1145/2817946.2817962

[17] Soroush Vosoughi, Deb Roy, and Sinan Aral. 2018. The spread of true and false news online. Science 359, 6380 (2018), 1146-1151. 